\title{
Chemical abundances for the transiting planet host stars OGLE-TR-10, 56, 111, 113, 132, and TrES-1 ${ }^{\star}, \star \star$
}

\section{Abundances in different galactic populations}

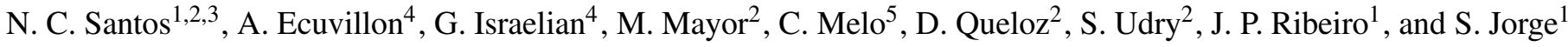 \\ 1 Centro de Astronomia e Astrofísica da Universidade de Lisboa, Observatório Astronómico de Lisboa, Tapada da Ajuda, \\ 1349-018 Lisboa, Portugal \\ e-mail: nuno@oal.ul.pt \\ 2 Observatoire de Genève, 51 Ch. des Maillettes, 1290 Sauverny, Switzerland \\ 3 Centro de Geofisica de Évora, Rua Romão Ramalho 59, 7002-554 Évora, Portugal \\ 4 Instituto de Astrofísica de Canarias, 38200 La Laguna, Tenerife, Spain \\ 5 European Southern Observatory, Casilla 19001, Santiago 19, Chile
}

Received 24 May 2006 / Accepted 22 June 2006

\section{ABSTRACT}

\begin{abstract}
Aims. We used the UVES spectrograph (VLT-UT2 telescope) to obtain high-resolution spectra of 6 stars hosting transiting planets, namely for OGLE-TR-10, 56, 111,113, 132, and TrES-1. These spectra are now used to derive and discuss the chemical abundances for $\mathrm{C}, \mathrm{O}, \mathrm{Na}, \mathrm{Mg}, \mathrm{Al}, \mathrm{Si}, \mathrm{S}, \mathrm{Ca}, \mathrm{Sc}, \mathrm{Ti}, \mathrm{V}, \mathrm{Cr}, \mathrm{Mn}, \mathrm{Co}, \mathrm{Ni}, \mathrm{Cu}$, and $\mathrm{Zn}$.

Methods. Abundances were derived in LTE, using 1D plane-parallel Kurucz model atmospheres. For S, Zn, and Cu, we used a spectral synthesis procedure, while for the remaining cases the abundances were derived from measurements of line-equivalent widths. Results. The resulting abundances were compared with those found for stars in the solar neighborhood. Distances and galactic coordinates were estimated for the stars. We conclude that, besides being particularly metal-rich and with small possible exceptions, OGLE-TR-10, 56, 111, 113, 132, and TrES-1 are chemically undistinguishable from the field (thin disk) stars regarding their [X/Fe] abundances. This is particularly relevant for the most distant of the targets, located up to $\sim 2 \mathrm{kpc}$ from the Sun. We also did not find any correlation between the abundances and the condensation temperature of the elements, evidence that strong accretion of planetary-like material, tentatively connected to planetary migration, did not occur.
\end{abstract}

Key words. stars: abundances - stars: fundamental parameters - stars: planetary systems - Galaxy: abundances stars: solar neighbourhood

\section{Introduction}

The discovery of several short period, transiting giant extrasolar planets is giving astronomers the possibility of measuring physical variables like the planetary radius, mass, and mean density. In all, 10 transiting cases have been discovered. While some of them are the outcome of the radial-velocity surveys (Charbonneau et al. 2000; Henry et al. 2000; Sato et al. 2005; Bouchy et al. 2005b), a few others were discovered in the context of photometric transit searches (Konacki et al. 2003; Bouchy et al. 2004; Pont et al. 2004; Alonso et al. 2004; Bouchy et al. 2005a; Konacki et al. 2005; McCullough et al. 2006).

The most prolific search for planetary transits has been carried out by the OGLE team (e.g. Udalski et al. 2002). After follow-up radial-velocity obervations, 5 of the more than 170 initial candidates were confirmed as real planetary transits. Unfortunately, all the OGLE-transiting planets orbit faint $(V$ 16) distant stars $(>500 \mathrm{pc})$, making it difficult to derive accurate stellar parameters and chemical abundances.

\footnotetext{
* Based on observations collected at the ESO 8.2-m VLT-UT2 Kueyen telescope (programs 075.C-0185 and 076.C-0131)

$\star \star$ Full Table 2 is only available in electronic form at the CDS via anonymous ftp to cdsarc.u-strasbg.fr $(130.79 .128 .5)$ or via http://cdsweb.u-strasbg.fr/cgi-bin/qcat?J/A+A/458/997
}

Recently, we obtained high-resolution and high signal-tonoise spectra of the 5 confirmed OGLE planet-host stars using the UVES spectrograph at the 8.2-m VLT/Kueyen telescope (ESO, Chile). These spectra were used to derive accurate stellar parameters for the stars, which were then used to improve the estimates of the planetary radii and mean-densities. The results for OGLE-TR-10, 56, 111, and 113, as well as for the brighter TrES1, were published in Santos et al. (2006). For OGLE-TR-132, the analysis is presented in Pont et al. (2006, in prep.). In a separate paper (Melo et al. 2006), we further discuss the ages of these stars, showing that they are older than $\sim 0.5-2$ Gyr. This conclusion has a strong impact on the evaporation rates of hot and very-hot jupiters. Finally, in Guillot et al. (2006) we show that a correlation between the stellar metallicity and the planetary internal structure seems to exist. If real, this result will have a strong impact on the models of planet formation.

In the current paper we present the abundances for several chemical elements in the 6 stars mentioned above (OGLE-TR$10,56,111,113,132$, and TrES-1). Using the derived stellar parameters and observed magnitudes, together with an estimate for the magnitude extinction, we derive the distances to the targets and their galactic coordinates. The results are then discussed and the abundances compared with those found for solar-neighborhood disk dwarfs. Finally, we compare the 
Table 1. Stellar parameters and metallicities for the 6 stars studied in this paper.

\begin{tabular}{lccccccl}
\hline \hline Star & $T_{\text {eff }}[\mathrm{K}]$ & $\log g$ (c.g.s.) & $\xi_{\mathrm{t}}\left[\mathrm{km} \mathrm{s}^{-1}\right]$ & {$[\mathrm{Fe} / \mathrm{H}]$} & $N(\mathrm{Fe}$ I, Fe II $)$ & $\sigma(\mathrm{Fe}$ I,Fe II) & Source \\
\hline OGLE-TR-10 & $6075 \pm 86$ & $4.54 \pm 0.15$ & $1.45 \pm 0.14$ & $0.28 \pm 0.10$ & 33,11 & $0.08,0.06$ & Santos et al. (2006) \\
OGLE-TR-56 & $6119 \pm 62$ & $4.21 \pm 0.19$ & $1.48 \pm 0.11$ & $0.25 \pm 0.08$ & 31,9 & $0.06,0.08$ & Santos et al. (2006) \\
OGLE-TR-111 & $5044 \pm 83$ & $4.51 \pm 0.36$ & $1.14 \pm 0.10$ & $0.19 \pm 0.07$ & 31,7 & $0.07,0.18$ & Santos et al. (2006) \\
OGLE-TR-113 & $4804 \pm 106$ & $4.52 \pm 0.26$ & $0.90 \pm 0.18$ & $0.15 \pm 0.10$ & 30,5 & $0.10,0.09$ & Santos et al. (2006) \\
OGLE-TR-132 & $6210 \pm 59$ & $4.51 \pm 0.27$ & $1.23 \pm 0.09$ & $0.37 \pm 0.07$ & 30,8 & $0.05,0.10$ & Pont et al. (2006, in prep.) \\
TrES-1 & $5226 \pm 38$ & $4.40 \pm 0.10$ & $0.90 \pm 0.05$ & $0.06 \pm 0.05$ & 36,7 & $0.04,0.05$ & Santos et al. (2006) \\
\hline
\end{tabular}

abundances of volatile and refractory elements in the 6 stars to look for possible evidence of the accretion of planetary material.

\section{Observations}

The observations were carried out with the UVES spectrograph at the VLT-UT2 Kueyen telescope. For TrES-1, OGLE-TR-10, 56, 111, and 113, the data were obtained between April and May 2005 in service mode (program ID 075.C-0185). More details are presented in Santos et al. (2006).

For OGLE-TR-132, we obtained 8 exposures of $3000 \mathrm{sec}-$ onds each (program ID 076.C-0131). The observations were done in December 2005 and January 2006. Each individual spectrum was then combined using the IRAF ${ }^{1}$ scombine routine. The total S/N obtained is close to 100, as measured directly from small spectral windows with no clear spectral lines in the region near $6500 \AA$. As for the other faint OGLE stars, the CCD was read in $2 \times 2$ bins for each exposure on OGLE-TR-132 to reduce the readout noise and increase the number of counts in each bin. This procedure does not compromise the resolving power, since the sampling of the CCD is still higher (by a factor of 2) than the instrumental PSF. Similarly we opted for using a slit width of 0.9 arcsec, which provides a spectral resolution $R=\lambda / \Delta \lambda \sim 50000$. The observations were made using the Dichroic 390+580 mode. The red portion of the spectra (used in this paper) covers the wavelength domain between 4780 and $6805 \AA$, with a gap between 5730 and $5835 \AA$.

As before, particular attention was paid to the orientation of the slit due to the relatively crowded field. The angle was chosen using the images available at the OGLE website ${ }^{2}$, so that no other star was present in the UVES slit during the observation.

\section{Stellar parameters and iron abundances}

Stellar parameters and iron abundances for our targets were taken from Santos et al. (2006) for TrES-1, OGLE-TR-10, 56, 111, and 113, and from Pont et al. (2006, in prep.) for OGLETR-132. These were derived in LTE using the 2002 version of the code MOOG (Sneden 1973) ${ }^{3}$ and a grid of Kurucz Atlas plane-parallel model atmospheres (Kurucz 1993). The whole procedure is described in Santos et al. (2004, and references therein) and is based on the analysis of $39 \mathrm{Fe} \mathrm{I}$ and $12 \mathrm{Fe}$ II weak lines and on imposing excitation and ionization equilibrium. Details about the analysis of the 6 stars in our sample are presented in Santos et al. (2006) and Pont et al. (2006, in prep.). The stellar parameters used in the current paper are summarized in Table 1.

\footnotetext{
${ }^{1}$ IRAF is distributed by National Optical Astronomy Observatories, operated by the Association of Universities for Research in Astronomy, Inc.,under contract with the National Science Foundation, USA.

${ }^{2}$ http://www.astrouw.edu.pl/ ftp/ogle/index.html

${ }^{3}$ http://verdi.as.utexas.edu/moog.html
}

Table 2. Line list used in the current paper for $\mathrm{Na}, \mathrm{Mg}, \mathrm{Al}, \mathrm{Si}, \mathrm{Ca}, \mathrm{Sc}$, $\mathrm{Ti}, \mathrm{V}, \mathrm{Cr}, \mathrm{Mn}, \mathrm{Co}$, and $\mathrm{Ni}$. The full table is available in electronic form at CDS.

\begin{tabular}{ccc}
\hline \hline$\lambda$ & $\chi_{l}$ & $\log g f$ \\
\hline Si I & & \\
5665.56 & 4.92 & -2.0000 \\
5690.43 & 4.93 & -1.8239 \\
5701.10 & 4.93 & -2.0372 \\
$\ldots$ & $\ldots$ & $\ldots$ \\
\hline
\end{tabular}

It is useful to mention that the stellar parameters used for the OGLE stars and TrES-1 were derived using the same methodology as the ones used by Bodaghee et al. (2003), Gilli et al. (2006), and Beirão et al. (2005) in their analysis of the same elements in the field-star samples mentioned below. This gives us a guarantee of uniformity in the comparison presented in Sect. 6.

\section{Abundances for other elements}

The analysis was done in LTE using a grid of Kurucz (1993) ATLAS-9 model atmospheres, and the 2002 version of the code MOOG (Sneden 1973). The abundances for $\mathrm{Na}, \mathrm{Mg}, \mathrm{Al}, \mathrm{Si}, \mathrm{Ca}$, $\mathrm{Sc}, \mathrm{Ti}, \mathrm{V}, \mathrm{Cr}, \mathrm{Mn}, \mathrm{Co}$, and $\mathrm{Ni}$ were derived from the analysis of line-equivalent widths (EW), measured by Gaussian fitting using the splot routine in IRAF. To derive the abundances we followed the procedure described in Beirão et al. (2005) for the first three elements of this series and in Bodaghee et al. (2003) and Gilli et al. (2006) for the remaining cases.

For the 12 elements listed above, we used the abfind driver in MOOG to find the abundances for each measured line giving as input the model atmosphere interpolated to the correct stellar parameters listed in Table 1, the measured EW, and the atomic parameters provided in Table 2 . The line-list we used is an upgraded version of the one used in Gilli et al. (2006), where we added a few more lines (except for $\mathrm{Na}, \mathrm{Mg}$, and $\mathrm{Al}$ ). Semiempirical $\log g f$ values were derived, as before, using equivalent widths obtained in the High Resolution Solar Atlas (Kurucz et al. 1984) and a solar model atmosphere with $T_{\text {eff }}=5777 \mathrm{~K}$, $\log g=4.44 \mathrm{dex}$, and $\xi_{t}=1.00 \mathrm{~km} \mathrm{~s}^{-1}$. The solar abundances were taken from Anders \& Grevesse (1989), except for iron, where the value of $\log \epsilon(\mathrm{Fe})=7.47$ was considered, as in all our previous studies. In our analysis of $\mathrm{Na}, \mathrm{Mg}, \mathrm{Al}, \mathrm{Si}, \mathrm{Ca}, \mathrm{Sc}$, $\mathrm{Ti}, \mathrm{V}, \mathrm{Cr}, \mathrm{Mn}, \mathrm{Co}$, and $\mathrm{Ni}$ we used the damping option " 2 " in MOOG.

We used both neutral (CrI and Ti I) and ionized (Cr II and Ti II) lines for $\mathrm{Cr}$ and $\mathrm{Ti}$. For the rest of the comparison, we use the abundances derived with the neutral lines, since the studies of the field stars were only based on the neutral species. A glance at Tables 3 and 4 shows that the two sets of lines generally yield similar results, within the errors.

Oxygen (O) abundances were derived from the forbiden [OI] line near $6300.3 \AA$. We followed the analysis procedure 


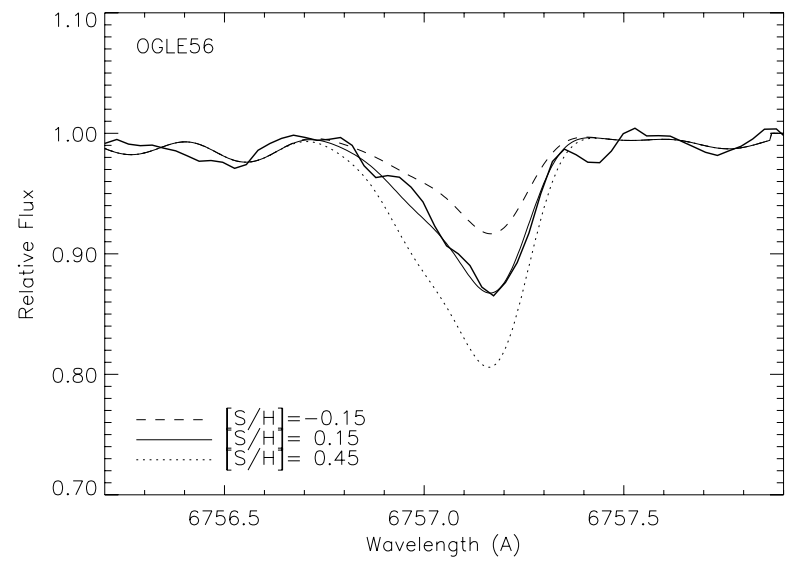

Fig. 1. Spectral synthesis of the S line at $6757.1 \AA$ for OGLE-TR-56. The solid line represents the observed spectrum, while three fits are denoted by the thinner lines.

described in detail in Ecuvillon et al. (2006b). Given that in metal-rich stars the [OI] line is blended with a smaller nickel line (e.g. Allende Prieto et al. 2001), we computed the expected EW for this latter line and subtracted it from the whole EW of the feature at $6300.3 \AA$. For OGLE-TR-113 and OGLE-TR-132, the quality of the spectra in the [OI] line region did not permit us to derive a quality value for the oxygen abundance. As in Ecuvillon et al. (2006b), we adopted a solar abundance of $\log \epsilon(\mathrm{O})=8.56$. In this case, similarly to $\mathrm{C}, \mathrm{S}, \mathrm{Zn}$, and $\mathrm{Cu}$ (below), we adopted the damping option " 0 " in MOOG (Unsold approximation).

For the abundances of carbon (C), sulphur $(\mathrm{S})$, zinc $(\mathrm{Zn})$, and copper $(\mathrm{Cu})$, we followed the prescription of Ecuvillon et al. (2004). Carbon abundances were derived using an EW analysis of two C I lines at 5380.3 and $5052.2 \AA$. Sulphur, zinc, and copper abundances were all obtained by spectral fitting to the data, using the synth driver in MOOG. We used two S I features near 6743.5 and $6757.1 \AA$ for $\mathrm{S}$. Zinc abundances were obtained from the $\mathrm{Zn}$ I line at $4810.5 \AA$, since the shorter wavelenth line at $4722.2 \AA$ used by Ecuvillon et al. (2004) is not present in our spectra. Finally, $\mathrm{Cu}$ abundances were derived from the analysis of the $\mathrm{Cu}$ I $5218.2 \AA$ line, since the feature at 5782.1 $\AA$ falls in the spectral gap of the UVES spectra. In the spectral synthesis, we considered a Gaussian broadening to take the instrumental profile into account. When available from the literature (Alonso et al. 2004; Pont et al. 2004; Bouchy et al. 2004, 2005a), the projected rotational velocity $v \sin i$ was also considered. When only an upper limit for the $v \sin i$ was available, we made use of other lines in the same spectral region to optimize the broadening function, following the same procedure as in Ecuvillon et al. (2004).

We refer to Ecuvillon et al. (2004) and Ecuvillon et al. (2006b) for the line-lists used and for more detail on the the derivation of the abundances of $\mathrm{C}, \mathrm{O}, \mathrm{S}, \mathrm{Zn}$, and $\mathrm{Cu}$. An example of the spectral synthesis for the derivation of $\mathrm{S}, \mathrm{Zn}$, and $\mathrm{Cu}$ abundances is presented in Figs. 1-3, respectively.

The final derived abundances are listed in Tables 3-6. In the first three, the uncertainties represent the rms around the average abundance for the cases where more than one line of the same element could be measured. For $\mathrm{C}, \mathrm{O}, \mathrm{S}, \mathrm{Zn}$, and $\mathrm{Cu}$, the final errors were computed adding the errors due to the uncertainties in atmospheric parameters in quadrature following the sensitivities listed in Ecuvillon et al. (2004) and Ecuvillon et al. (2006b), together with the uncertainty in the abundance synthesis or analysis. For this, the rms was used when more than one line was

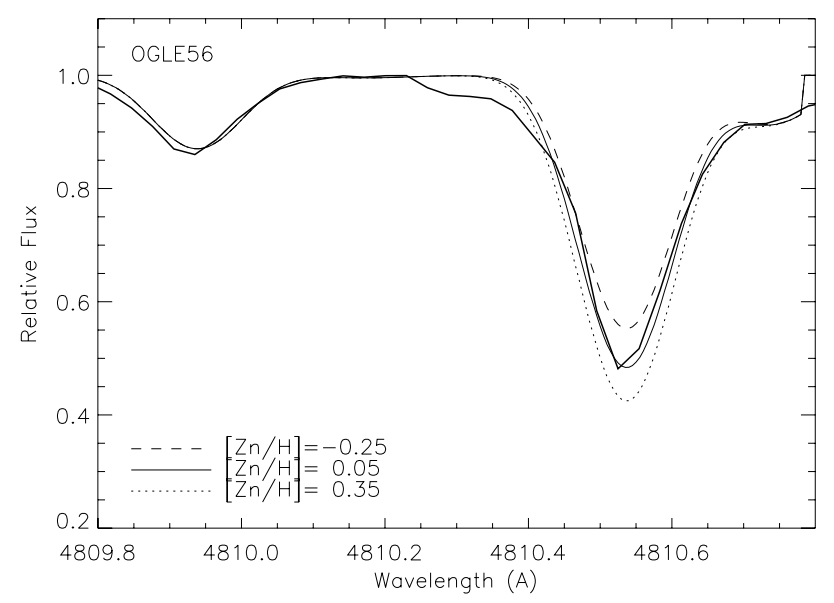

Fig. 2. Spectral synthesis of the Zn line at $4810.5 \AA$ for OGLE-TR-56.

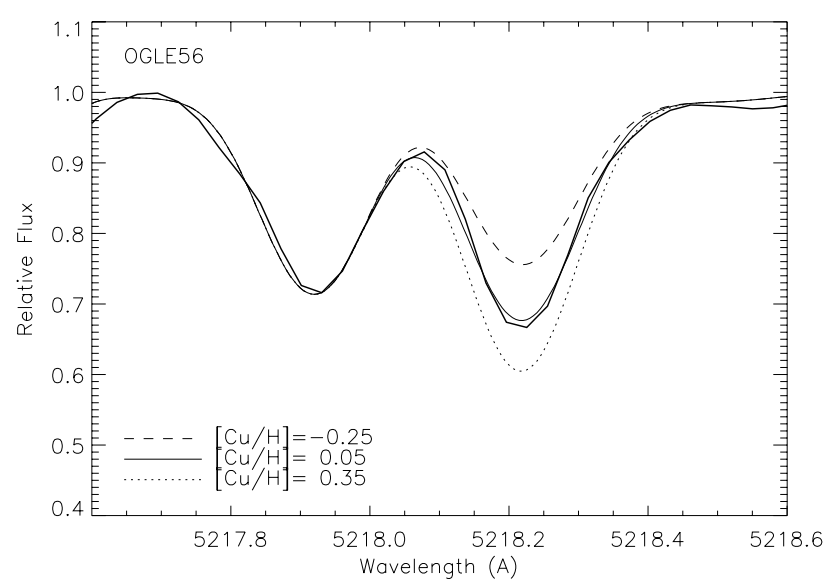

Fig. 3. Spectral synthesis of the $\mathrm{Cu}$ line at $5218.2 \AA$ for OGLE-TR-56.

measured; otherwise, the uncertainty in the measured EW was considered. For $\mathrm{S}, \mathrm{Zn}$, and $\mathrm{Cu}$, the uncertainty in the synthesis procedure was taken into account.

\subsection{Comparison with the literature}

The chemical abundances for the faint OGLE stars have not been studied elsewhere in the literature. However, for the brighter TrES-1, Sozzetti et al. (2006) have derived chemical abundances for a series of elements, most of which are also studied in this paper.

In general, the abundances derived by Sozzetti et al. (2006) for TrES-1 agree with the ones presented here. However, we do see a systematic difference between the two studies. On average, our values are slightly higher (by 0.08 dex, with a dispersion around the average value of 0.08 ) than the ones derived by the study of Sozzetti et al. This small systematic difference can be due to the use of slighly different atmospheric parameters and line-lists.

\section{Distances and galactic positions}

We used the available information regarding the 6 stars in our sample to derive their distances and galactic positions. Since most of the targets (all the OGLE stars) have only I magnitudes available, we decided to work in this band and derived the $I$ magnitude of TrES-1 by inverting the $T_{\text {eff }}:(V-I)$ calibration 
Table 3. Derived abundances for the alpha elements in the 6 stars studied in this paper. The errors denote the rms around the average abundance given by each of the $n(\mathrm{X})$ lines used for the element $\mathrm{X}$.

\begin{tabular}{|c|c|c|c|c|c|c|c|c|c|c|}
\hline Star & {$[\mathrm{Si} / \mathrm{H}]$} & $n(\mathrm{Si})$ & {$[\mathrm{Ca} / \mathrm{H}]$} & $n(\mathrm{Ca})$ & {$[\mathrm{TiI} / \mathrm{H}]$} & $n$ (TiI) & [Till/H] & $n$ (Till) & {$[\mathrm{Sc} / \mathrm{H}]$} & $n(\mathrm{Sc})$ \\
\hline OGLE-TR-10 & $0.32 \pm 0.04$ & 8 & $0.30 \pm 0.04$ & 10 & $0.31 \pm 0.05$ & 9 & $0.31 \pm 0.09$ & 4 & $0.33 \pm 0.10$ & 4 \\
\hline OGLE-TR-56 & $0.28 \pm 0.05$ & 9 & $0.26 \pm 0.08$ & 11 & $0.22 \pm 0.04$ & 6 & $0.29 \pm 0.05$ & 4 & $0.35 \pm 0.05$ & 6 \\
\hline OGLE-TR-111 & $0.10 \pm 0.04$ & 5 & $0.21 \pm 0.08$ & 9 & $0.35 \pm 0.07$ & 10 & $0.15 \pm 0.10$ & 4 & $0.11 \pm 0.13$ & 2 \\
\hline OGLE-TR-113 & $0.16 \pm 0.12$ & 7 & $0.00 \pm 0.07$ & 8 & $0.29 \pm 0.09$ & 13 & $0.20 \pm 0.17$ & 4 & $0.14 \pm 0.12$ & 4 \\
\hline OGLE-TR-132 & $0.36 \pm 0.03$ & 8 & $0.31 \pm 0.04$ & 12 & $0.35 \pm 0.07$ & 6 & $0.36 \pm 0.10$ & 4 & $0.49 \pm 0.03$ & 7 \\
\hline TrES-1 & $0.06 \pm 0.04$ & 9 & $0.05 \pm 0.07$ & 12 & $0.11 \pm 0.06$ & 13 & $0.00 \pm 0.01$ & 4 & $0.02 \pm 0.06$ & 5 \\
\hline
\end{tabular}

Table 4. Same as Table 3 for the iron-peak elements Mn, V, Cr and Co.

\begin{tabular}{|c|c|c|c|c|c|c|c|c|c|c|}
\hline Star & {$[\mathrm{Mn} / \mathrm{H}]$} & $n(\mathrm{Mn})$ & [V/H] & $n(\mathrm{~V})$ & {$[\mathrm{CrI} / \mathrm{H}]$} & $n(\mathrm{CrI})$ & {$[\mathrm{CrII} / \mathrm{H}]$} & $n(\mathrm{CrII})$ & {$[\mathrm{Co} / \mathrm{H}]$} & $n(\mathrm{Co})$ \\
\hline OGLE-TR-10 & $0.31 \pm 0.08$ & 3 & $0.31 \pm 0.07$ & 3 & $0.38 \pm 0.25$ & 2 & $0.32 \pm 0.01$ & 2 & $0.39 \pm 0.00$ & 1 \\
\hline OGLE-TR-56 & $0.33 \pm 0.16$ & 3 & $0.23 \pm 0.07$ & 4 & $0.32 \pm 0.00$ & 1 & $0.25 \pm 0.18$ & 2 & $0.25 \pm 0.11$ & 4 \\
\hline OGLE-TR-111 & $0.37 \pm 0.13$ & 3 & $0.69 \pm 0.14$ & 8 & $0.21 \pm 0.09$ & 4 & $-0.02 \pm 0.00$ & 1 & $0.30 \pm 0.10$ & 8 \\
\hline OGLE-TR-113 & $0.29 \pm 0.08$ & 2 & $0.56 \pm 0.16$ & 9 & $0.10 \pm 0.08$ & 4 & $0.19 \pm 0.00$ & 1 & $0.30 \pm 0.15$ & 7 \\
\hline OGLE-TR-132 & $0.37 \pm 0.12$ & 3 & $0.32 \pm 0.18$ & 4 & $0.25 \pm 0.03$ & 2 & $0.30 \pm 0.10$ & 3 & $0.34 \pm 0.09$ & 6 \\
\hline TrES-1 & $0.14 \pm 0.01$ & 3 & $0.21 \pm 0.07$ & 8 & $0.04 \pm 0.02$ & 4 & $0.00 \pm 0.00$ & 1 & $0.06 \pm 0.09$ & 7 \\
\hline
\end{tabular}

Table 5. Same as Table 3 for the iron-peak element $\mathrm{Ni}$, and for $\mathrm{Na}, \mathrm{Mg}$ and $\mathrm{Al}$.

\begin{tabular}{lcccccccc}
\hline \hline Star & {$[\mathrm{Ni} / \mathrm{H}]$} & $n(\mathrm{Ni})$ & {$[\mathrm{Na} / \mathrm{H}]$} & $n(\mathrm{Na})$ & {$[\mathrm{Mg} / \mathrm{H}]$} & $n(\mathrm{Mg})$ & {$[\mathrm{Al} / \mathrm{H}]$} & $n(\mathrm{Al})$ \\
\hline OGLE-TR-10 & $0.22 \pm 0.08$ & 21 & $0.24 \pm 0.11$ & 3 & $0.23 \pm 0.14$ & 3 & $0.29 \pm 0.00$ & 1 \\
OGLE-TR-56 & $0.27 \pm 0.06$ & 23 & $0.38 \pm 0.09$ & 3 & $0.22 \pm 0.17$ & 3 & $0.30 \pm 0.01$ & 2 \\
OGLE-TR-111 & $0.22 \pm 0.10$ & 22 & $0.21 \pm 0.17$ & 3 & $0.23 \pm 0.12$ & 3 & $0.28 \pm 0.15$ & 2 \\
OGLE-TR-113 & $0.17 \pm 0.10$ & 20 & $0.06 \pm 0.17$ & 3 & $0.22 \pm 0.18$ & 3 & $0.22 \pm 0.02$ & 2 \\
OGLE-TR-132 & $0.36 \pm 0.10$ & 22 & $0.31 \pm 0.02$ & 3 & $0.26 \pm 0.10$ & 2 & $0.40 \pm 0.03$ & 2 \\
TrES-1 & $0.06 \pm 0.04$ & 26 & $-0.05 \pm 0.06$ & 3 & $0.05 \pm 0.08$ & 3 & $0.10 \pm 0.01$ & 2 \\
\hline
\end{tabular}

Table 6. Same as Table 3 for C, O, S, Zn, and Cu. For Cu, in OGLE-TR-113, the abundance was obtained only from the $5380 \AA$ line; the uncertainty was derived based on an estimate of the measured EW. For TrES-1, only the 6757 Å line of sulphur was measured.

\begin{tabular}{lccccc}
\hline \hline Star & {$[\mathrm{C} / \mathrm{H}]$} & {$[\mathrm{O} / \mathrm{H}]$} & {$[\mathrm{S} / \mathrm{H}]$} & {$[\mathrm{Zn} / \mathrm{H}]$} & {$[\mathrm{Cu} / \mathrm{H}]$} \\
\hline OGLE-TR-10 & $0.29 \pm 0.09$ & $0.31 \pm 0.20$ & $0.13 \pm 0.12$ & $0.47 \pm 0.11$ & $0.27 \pm 0.10$ \\
OGLE-TR-56 & $0.24 \pm 0.14$ & $0.35 \pm 0.26$ & $0.20 \pm 0.09$ & $0.09 \pm 0.09$ & $0.23 \pm 0.08$ \\
OGLE-TR-111 & $0.43 \pm 0.21$ & $0.21 \pm 0.38$ & $<0.64$ & $0.13 \pm 0.11$ & $0.47 \pm 0.10$ \\
OGLE-TR-113 & $0.81 \pm 0.21$ & - & $<0.70$ & $0.34 \pm 0.11$ & $0.53 \pm 0.11$ \\
OGLE-TR-132 & $0.27 \pm 0.10$ & - & $0.42 \pm 0.11$ & $0.21 \pm 0.09$ & $0.30 \pm 0.09$ \\
TrES-1 & $0.06 \pm 0.07$ & $0.11 \pm 0.17$ & $<0.26$ & $0.05 \pm 0.06$ & $0.04 \pm 0.08$ \\
\hline
\end{tabular}

of Alonso et al. (1996). For this we considered $V=11.79$ and made use of the effective temperature listed in Table 1.

With the effective temperatures listed in the table and the best available stellar radii estimates (Pont et al. 2006, in prep.; Bouchy et al. 2004; Laughlin et al. 2005; Santos et al. 2006), we derived the stellar luminosities. We then used the $M_{\text {bol }}: L$ relation presented in Lang (1999) to obtain the bolometric magnitude of each star. From $M_{\text {bol }}$, the absolute magnitudes $\left(M_{I}\right)$ were derived with the bolometric correction for the $I$ band $\left(\mathrm{BC}_{I}\right)$ taken from Bessell et al. (1998). Finally, with $M_{I}$ and the apparent $I$ magnitude and with neglecting the interstellar absorption, we could derive a first guess for the stellar distances $\left(d_{0}\right)$.

Once a first value was available, we used a fortran version of the IDL code EXTIN. PRO (Amôres \& Lépine 2005) ${ }^{4}$ and the relation $A_{I} / A_{V} \sim 0.601$ (Schlegel et al. 1998) ${ }^{5}$ to obtain a value for $A_{V}$ and $A_{I}$. This was then used to obtain a new value for

\footnotetext{
${ }^{4}$ The code was kindly provided by the authors. The IDL version is available at http://www.astro.iag.usp.br/ jacques/ programs.html

5 Similar results are derived from the tables of Grebel \& Roberts (1995).
}

the stellar distance. All this procedure was followed iteractively, until the distances remained unchanged. The final values, as well as the first guess (considering null extinction), are presented in Table 7. The null-extinction value for TrES-1 is very close to the one derived in previous works (Alonso et al. 2004; Laughlin et al. 2005). For this star, a distance of $158 \mathrm{pc}$ is derived using the observed $V$ magnitude, the bolometric correction from Flower (1996) and considering zero reddening.

Once we had the distance to the stars and the $l, b$ galactic coordinates ${ }^{6}$ we obtained the $X, Y$, and $Z$ galactic positions for the 6 stars (Fig. 4, Table 7). As we can see from the figure, the OGLE stars in the different fields (Carina at $l \sim 289$ degrees and Bulge at $l \sim 0$ degrees) are all at very different distances from the Sun. Althought the Carina-field stars (OGLE-TR-111, 113, and 132) all have a similar galactocentric radius as the Sun, the same is not true for the Bulge-field stars (OGLE-TR-10 and 56). Here we consider that the Sun is at $R_{\mathrm{g}}=8.5 \mathrm{kpc}$.

${ }^{6}$ Derived using the epoch 2000.0 right-ascention and declinations and making use of the the galactic routine within the IRAF "astutils" package. 
Table 7. Stellar $I$ magnitudes, stellar radii, galactic coordinates, derived distances, $X, Y, Z, R_{\mathrm{g}}$ galactic coordinates, adopted interstellar extinction, and systemic radial-velocities for the 6 stars studied in this paper. The distance derived considering null extition, $d_{0}$, is also presented. For TrES-1, the $I$ magnitude was derived from the $V$ magnitude $(V=11.79)$ and the known effective temperature. See text for more details.

\begin{tabular}{|c|c|c|c|c|c|c|c|c|c|c|c|c|}
\hline Star & $\begin{array}{c}I \\
{[\mathrm{mag}]}\end{array}$ & $\begin{array}{l}R_{\text {star }} \\
{\left[R_{\odot}\right]}\end{array}$ & $\begin{array}{c}l \\
{\left[{ }^{\circ}\right]}\end{array}$ & $\begin{array}{c}b \\
{\left[{ }^{\circ}\right]}\end{array}$ & $\begin{array}{c}d_{0} \\
{[\mathrm{pc}]}\end{array}$ & $\left.\begin{array}{c}d \\
{[\mathrm{pc}]}\end{array}\right]$ & $\begin{array}{c}X \\
{[\mathrm{pc}]}\end{array}$ & $\left.\begin{array}{c}Y \\
{[\mathrm{pc}]}\end{array}\right]$ & $\begin{array}{c}Z \\
{[\mathrm{pc}]}\end{array}$ & $\begin{array}{c}R_{\mathrm{g}} \\
{[\mathrm{pc}]}\end{array}$ & $\begin{array}{c}A v \\
{[\mathrm{mag}]}\end{array}$ & $\begin{array}{c}R V \\
{\left[\mathrm{~km} \mathrm{~s}^{-1}\right]}\end{array}$ \\
\hline OGLE-TR-10 & 14.9 & 1.14 & 359.8516 & -1.5767 & 1830 & 1326 & -3 & 7174 & -36 & 7175 & 1.16 & $-6.2^{a}$ \\
\hline OGLE-TR-56 & 15.3 & 1.15 & 0.7053 & -2.3655 & 2246 & 1591 & 20 & 6911 & -66 & 6911 & 1.25 & $-48.3^{a}$ \\
\hline OGLE-TR-111 & 15.5 & 0.83 & 289.2794 & -1.7056 & 1236 & 1011 & -954 & 8166 & -30 & 8222 & 0.72 & $25.1^{b}$ \\
\hline OGLE-TR-113 & 14.4 & 0.765 & 289.2017 & -1.7895 & 614 & 553 & -522 & 8318 & -17 & 8335 & 0.42 & $-7.9^{c}$ \\
\hline OGLE-TR-132 & 15.7 & 1.28 & 289.2349 & -2.3430 & 3081 & 2180 & -2057 & 7782 & -89 & 8050 & 1.25 & $39.7^{c}$ \\
\hline TrES-1 & 10.73 & 0.83 & 67.4649 & 13.4403 & 148 & 143 & 129 & 8447 & 33 & 8448 & 0.11 & $-20.7^{d}$ \\
\hline
\end{tabular}

${ }^{a}$ Bouchy et al. (2005a); ${ }^{b}$ Pont et al. (2004); ${ }^{c}$ Bouchy et al. $(2004) ;{ }^{d}$ this paper.

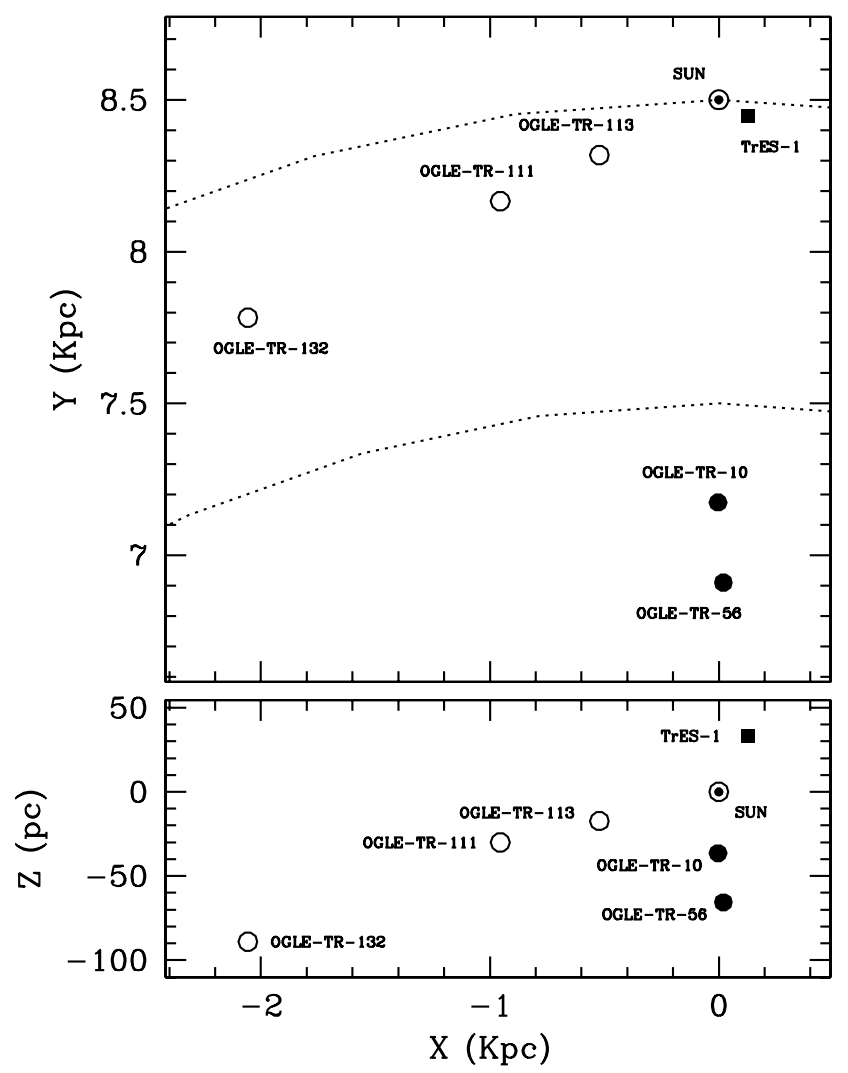

Fig. 4. Galactic Cartesian $X, Y$, and $Z$ coordinates of our stars and the Sun. The coordinate system is right-handed. Here we considered that the Sun is at a galactocentric radius of $8.5 \mathrm{kpc}$ in the $Y$ direction. Different symbols are considered for stars in the different fields: OGLETR-10 and 56 (filled circles), OGLE-TR-111, 113, and 132 (open circles), and TrES-1 (filled square). The dotted curves represent the location of the 8.5 and $7.5 \mathrm{kpc}$ galactocentric regions. In this figure we are basically representing the 4 th galactic quadrant.

We should add that errors in the stellar radii, magnitudes, and temperatures, together with possibly large uncertainties in the derivation of the interstellar reddening, imply that the values listed in Table 7 must be seen as indicative.

\section{Galactic chemical trends}

The use of chemical abundances to distinguish between different populations in the Galaxy (in particular the thin disk, thick disk, and galactic bulge) has been thoroughly discussed in the literature (e.g. Bensby et al. 2003; Nissen 2004; Fuhrmann 2004; Brewer \& Carney 2006; Fulbright et al. 2005). The observed differences are meant to reflect different star formation histories in the different galactic systems. It is thus interesting to check whether the abundances of the elements studied in the current paper for TrES-1 and the 5 OGLE stars differ from the ones measured in field disk stars.

In Figs. 5-8 we present the $[\mathrm{X} / \mathrm{Fe}]$ vs. $[\mathrm{Fe} / \mathrm{H}]$ plots for all the elements studied in the current paper. In the figures, the data regarding the solar-neighborhood field stars (planet-hosts and "single" stars) was taken from Bodaghee et al. (2003), Beirão et al. (2005), and Gilli et al. (2006) for $\mathrm{Na}, \mathrm{Mg}, \mathrm{Al}, \mathrm{Si}, \mathrm{Ca}, \mathrm{Sc}, \mathrm{Ti}$, $\mathrm{V}, \mathrm{Cr}, \mathrm{Mn}, \mathrm{Co}$, and Ni, and from Ecuvillon et al. (2004, 2006b) for $\mathrm{O}, \mathrm{C}, \mathrm{S}, \mathrm{Cu}$, and $\mathrm{Zn}$. The choice of these samples is based on the fact that these were studied using the same line-lists, analysis methods, and model atmospheres as used in the current study, making us confident that a comparison will be unbiased. In the figure, the 6 stars studied in this paper are denoted by the larger symbols. The choice of the different symbols (explained in the figure caption) is important since these stars belong to different populations in the Galaxy (see Sect. 5).

As seen from the plots, the 5 OGLE stars and TrES-1 are chemically indistinguishable in a first analysis from the field stars used as reference (mostly thin disk stars - Ecuvillon et al., in preparation), except for the fact that they represent a particularly metal-rich "population". No major differences are found for any of the elements studied. In particular, no important alphaelement enhancement is observed. Such a trend could be typical of galactic bulge stars (e.g. Fulbright et al. 2005) or of thick-disk objects (see Fuhrmann 2004). The radial-velocities presented in Table 7 also do not show any particular anomally. These same conclusions were also reached by Sozzetti et al. (2006) concerning TrES-1.

This result is probably not unexpected for the closest stars in the Carina field (OGLE-TR-111,113), all with galactocentric radii similar to the Sun. However, some differences could be expected for OGLE-TR-10 and 56, located at $\sim 1.5 \mathrm{kpc}$ in the direction of the galactic center, and for OGLE-TR-132, at more than $2 \mathrm{kpc}$ from us. The fact that the studied stars are not young (Melo et al. 2006), together with the stellar galactic velocity dispersion (Nordström et al. 2004), may help to explain this similarity.

A careful look at the plots shows, however, that some small differences may exist. For instance, OGLE-TR-132 generally seems to occupy a position in the lower envelope of the points in the field star distribution. Given that this systematic effect is observed for most of the elements, this difference, although small, seems to be real. Interestingly, according to the calculations in Sect. 5, this star is the most distant of all the targets studied here.

Large differences may also be found for OGLE-TR-111 and 113 regarding $\mathrm{V}, \mathrm{C}, \mathrm{Cu}$, and maybe $\mathrm{Ti}$, where these two stars 

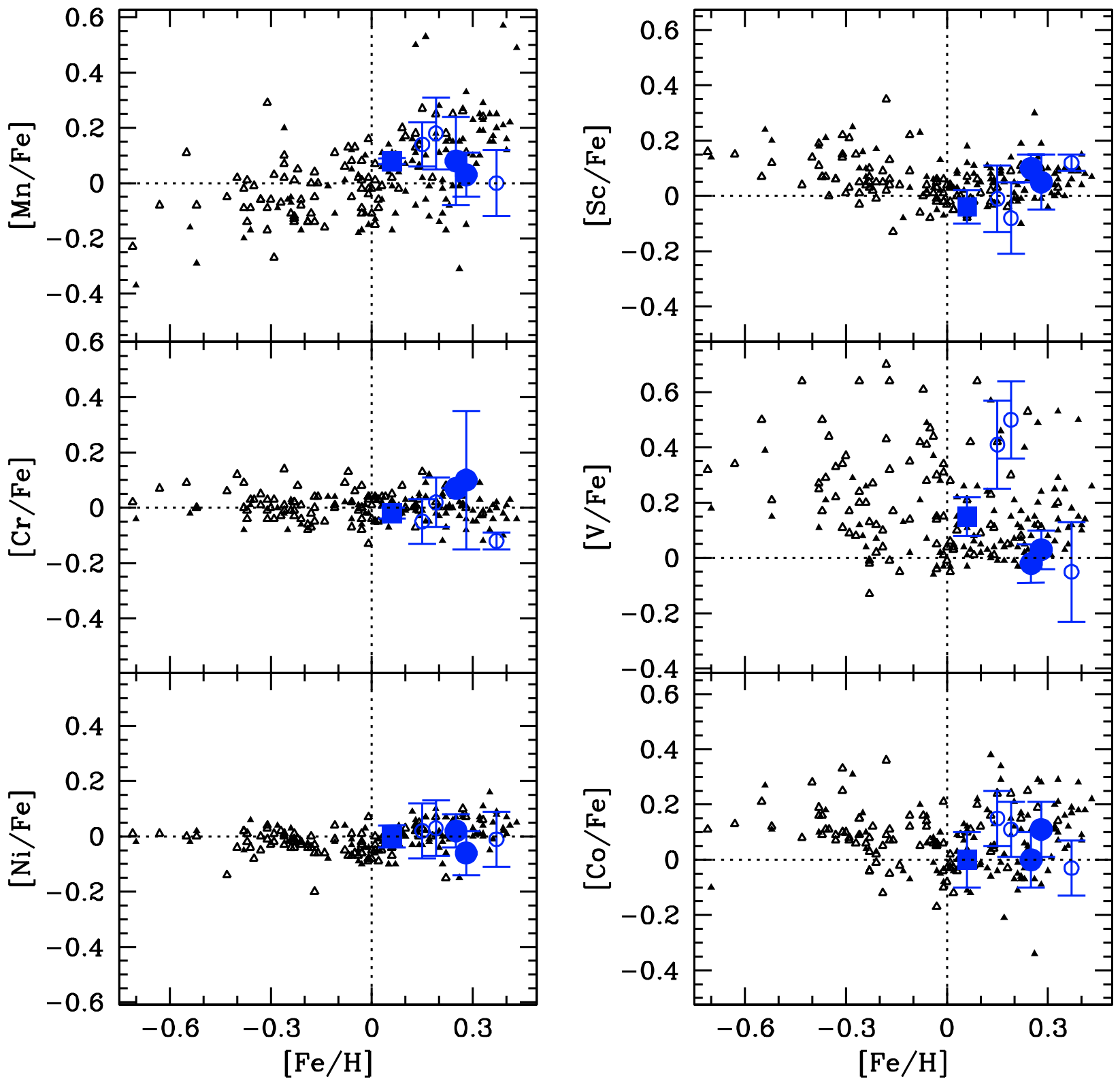

Fig. 5. $[\mathrm{X} / \mathrm{Fe}]$ vs. $[\mathrm{Fe} / \mathrm{H}]$ plots for $\mathrm{X}=\mathrm{Ni}, \mathrm{Cr}, \mathrm{Mn}, \mathrm{Co}, \mathrm{V}$ (Fe-peek elements), and $\mathrm{Sc}$ (alpha-element). Filled triangles represent stars with giant planets, and open triangles denote single field stars. The other symbols represent OGLE-TR-10 and 56 (filled circles), OGLE-TR-111, 113, and 132 (open circles), and TrES-1 (filled square). The error bars represent the rms around the average abundance when more than one line was used to derive the chemical abundances. When one single line was used, we adopted an error of 0.10 dex in these plots.

seem to be particularly overabundant. However, we should mention that the observed differences for these elements may be due to analysis effects. OGLE-TR-111 and 113 are the two coolest stars in our sample. As shown by Bodaghee et al. (2003), V and $\mathrm{Ti}$ are among the elements presenting the strongest $[\mathrm{X} / \mathrm{Fe}]$ vs. $T_{\text {eff }}$ trends, possibly due to NLTE effects, where the lowest temperature stars present higher abundances. The high abundances of $\mathrm{C}$ and $\mathrm{Cu}$ are more difficult to explain, as no clear dependence of the derived abundances on the effective temperature was found for these two elements in the study of Ecuvillon et al. (2004). The very high carbon abundance of OGLE-TR-113 is particularly intriguing, although the derived value is based only on one of the two available carbon lines.

The abundances of $\mathrm{Cu}$ for TrES-1, OGLE-TR-10, and 56 are a bit below the field star sample, although in agreement within the errors. A similar residual low value is found for $\mathrm{Al}$ and most clearly for $\mathrm{Mg}$ in these stars. Finally, the $\mathrm{Ca}$ abundance for OGLE-TR-113 seems to be particularly low, although the other alpha elements do not show the same tendency.

\section{Condensation temperature}

The accretion of planetary material has been proposed by several authors to explain the high metal content observed in stars orbited by giant planets (e.g. Gonzalez 1998; Murray et al. 2002). This effect could be particularly important concerning the stars with very short-period planets, since planetary migration could increase the quantity of planetesimals falling onto the stellar surface (Murray et al. 1998).

Abundance traces of pollution events in the atmospheres of stars with giant planets have indeed been reported in the literature in some particular cases (Israelian et al. 2001; 

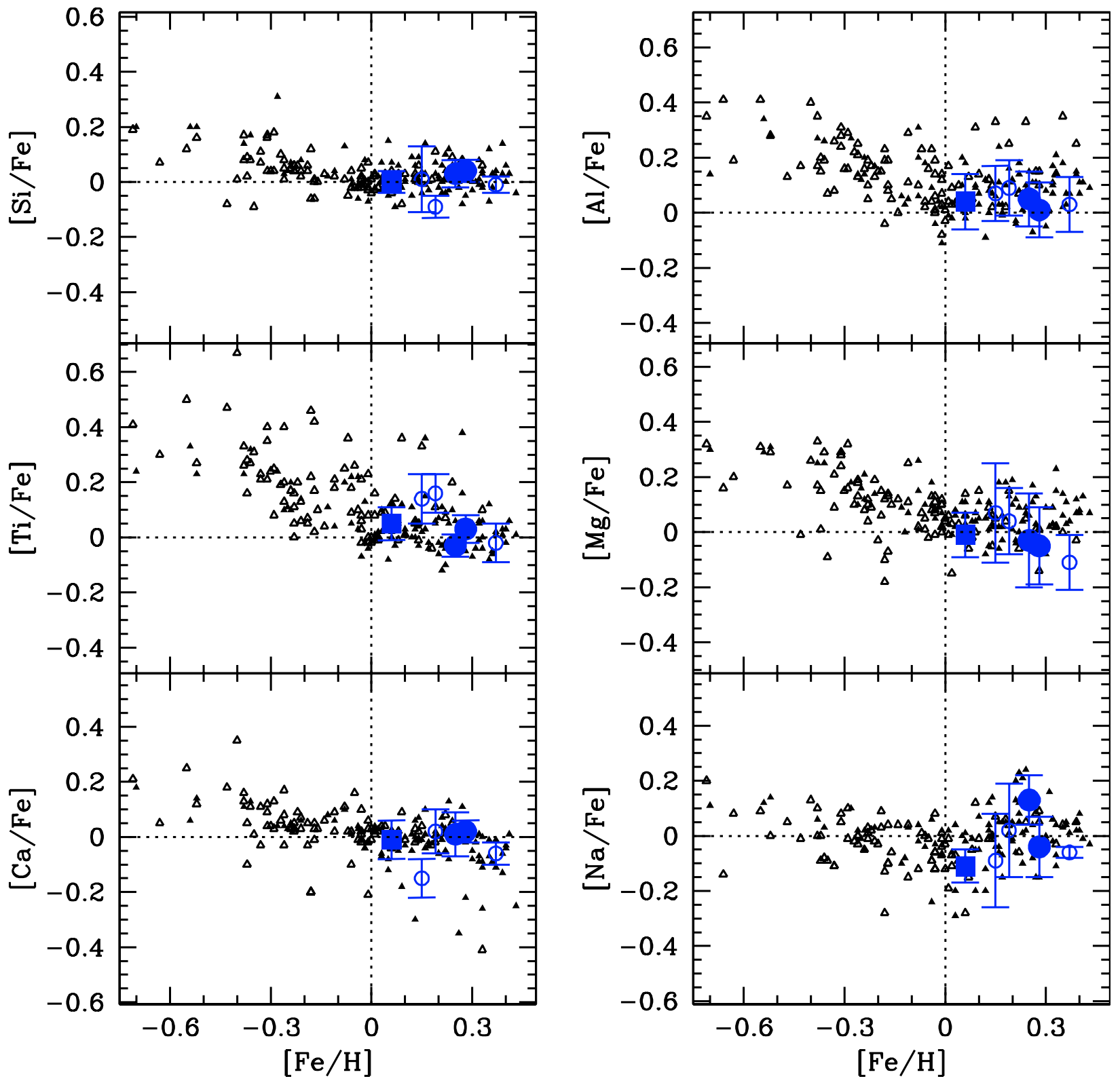

Fig. 6. Same as Fig. 5 for $\mathrm{X}=\mathrm{Ca}, \mathrm{Ti}, \mathrm{Si}$ (alpha-elements), $\mathrm{Na}, \mathrm{Mg}$, and $\mathrm{Al}$.

Laws \& Gonzalez 2001). Although several observational results have made this hypothesis unlikely (e.g. Pinsonneault et al. 2001; Santos et al. 2003; Fischer \& Valenti 2005), even if they did not completely discard it (e.g. Vauclair 2004), it is interesting to explore this possibility in our case, since all 6 stars in our sample are both metal-rich and orbited by short period giant planets.

If a solar-type star engulfs a significant amount of planetarylike material, we can expect to observe that some elements have been enriched more than others. Such an effect is expected since lower condensation temperature elements will probably evaporate before falling into the star. This idea may be valid, however, only if the infall of material is slow enough or if the infalling bodies are not too big, so that the low condensation temperature material is able to escape. Having this possibility in mind, several authors have studied the trends in element abundance as a function of the condensation temperature of the elements in stars with giant planets (Smith et al. 2001; Takeda et al. 2001; Sadakane et al. 2002; Ecuvillon et al. 2006a).
Using the chemical abundances listed in Tables 1 through 6 , we derived the slopes of the relation between $[\mathrm{X} / \mathrm{H}]$ and $T_{\text {cond }}$ for the stars in our sample (Fig. 9). We used the same methodology as in Ecuvillon et al. (2006a), making use of the condensation temperatures for the elements listed in Lodders (2003). In our case, these go from $78 \mathrm{~K}$ for carbon to $1677 \mathrm{~K}$ for aluminum.

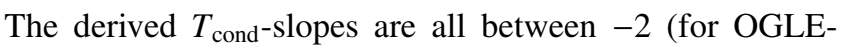
TR-111) and $6 \mathrm{dex} / 100000 \mathrm{~K}$ (for OGLE-TR-132), well within the values obtained for the other stars in the Ecuvillon et al. (2006a) sample. The only exception is OGLE-TR-113, whose

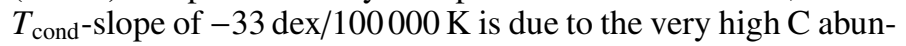
dance observed for this star. Excluding the carbon abundances for this star, the slope obtained is only of $-5 \mathrm{dex} / 100000 \mathrm{~K}$. Unfortunately, in this particular case the abundance of carbon is based on one single carbon line, so we cannot confirm the observed $\mathrm{C}$ abundance. We note, however, that a negative slope indicates a low refractory-to-volatile ratio, contrary to what would be expected if pollution were responsible for any element abundance changes. 

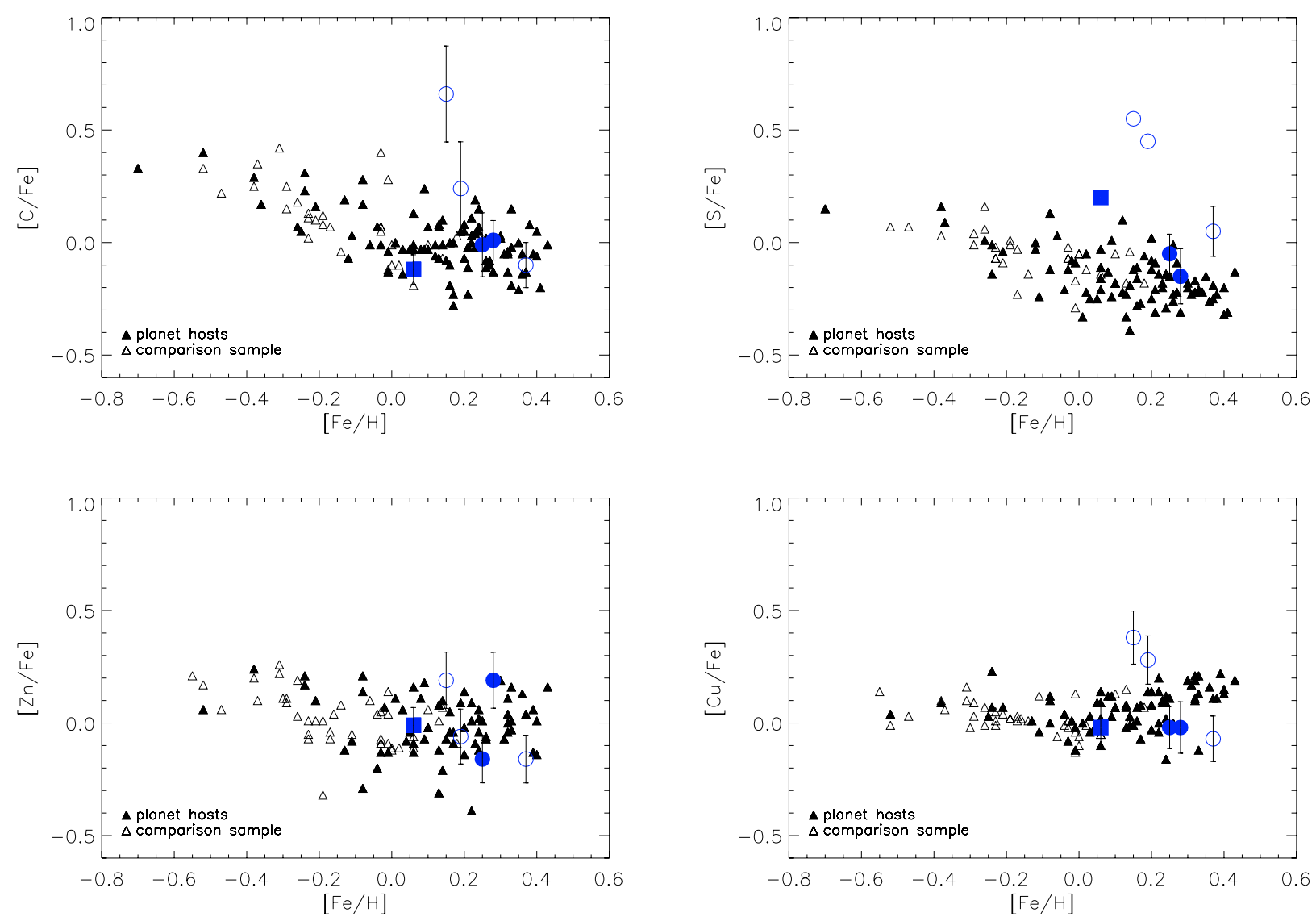

Fig. 7. Same as Fig. 5 for $\mathrm{X}=\mathrm{C}, \mathrm{S}, \mathrm{Zn}$, and $\mathrm{Cu}$. For S, the points of TrES-1, OGLE-TR-111, and 113 (without error bars) represent upper limits on the abundances.

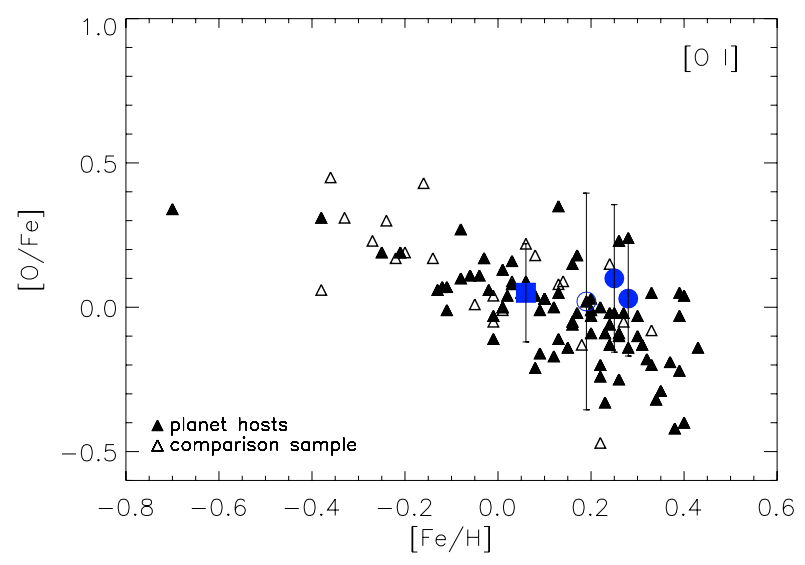

Fig. 8. Same as Fig. 5 for oxygen.

In all, we do not find any clear evidence for differential accretion in the 6 planet-host stars analyzed.

\section{Concluding remarks}

We have derived the abundances of $\mathrm{C}, \mathrm{O}, \mathrm{Na}, \mathrm{Mg}, \mathrm{Al}, \mathrm{Si}, \mathrm{S}, \mathrm{Ca}$, $\mathrm{Sc}, \mathrm{Ti}, \mathrm{V}, \mathrm{Cr}, \mathrm{Mn}, \mathrm{Co}, \mathrm{Ni}, \mathrm{Cu}$, and $\mathrm{Zn}$ in the transiting planethost stars OGLE-TR-10, 56, 111, 113, 132, and TrES-1. Using the available planetary radii and effective temperatures, as well as a model for the interstellar extition, we also derived the galactic positions of the 6 stars. The results show that OGLE-TR-10 and 56 are more than $1 \mathrm{kpc}$ inside the solar radius, while the
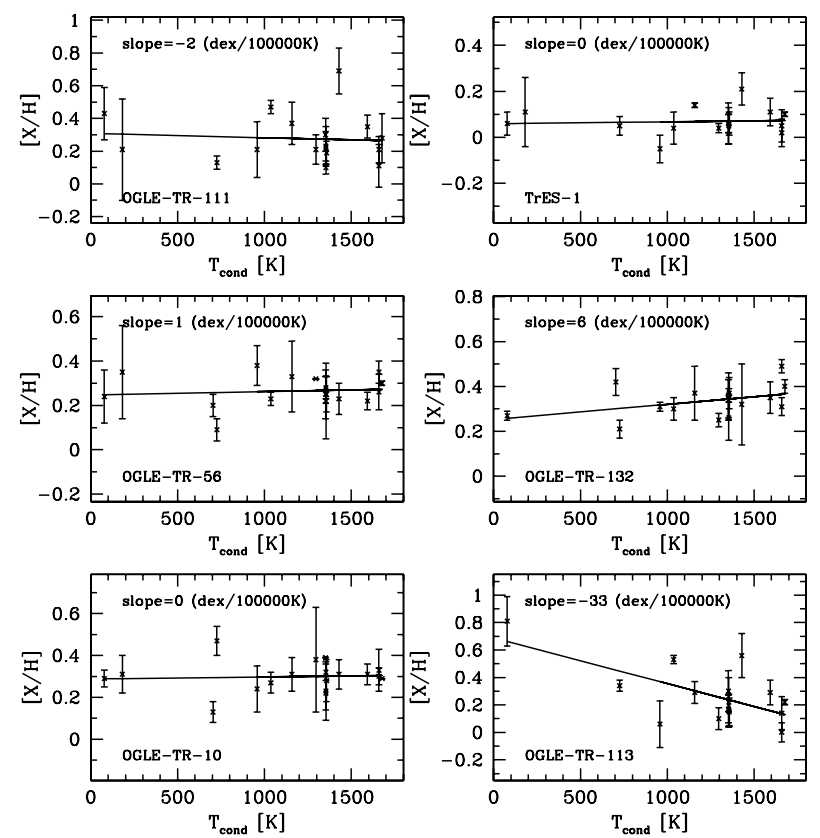

Fig. 9. Element abundances as a function of the condensation temperature for the 6 stars studied in the current paper. The measured slopes are shown in each panel.

remaining stars have similar galactocentric radius as the Sun, although at a variety of distances.

A comparison of the chemical abundances with the ones found for stars in the solar neighborhood shows that apart 
from the fact that they are particularly metal-rich, no major differences exist for the relative element-over-iron abundances. Given their galactic positions, this result is probably not unexpected for OGLE-TR-111, 113, and TrES-1, but it may be particularly interesting for the cases of OGLE-TR-10, 56, and 132.

Using the obtained chemical abundances and the condensation temperatures of the elements, we then explored the possibility that the 6 stars, all orbited by short period giant planets, may have accreted planetary-like material. The results show no clear evidence of this, giving stronger support to the idea that the metal content observed in the stars with giant planets is not due to the accretion of metal-rich, hydrogen-poor material.

Acknowledgements. We would like to thank A. Moitinho, S. Sousa, J. Lepine, and E. Amores for their help in deriving the interstellar extinction values. Support from the Fundação para a Ciência e a Tecnologia (Portugal) to N.C.S in the form of a fellowship (reference SFRH/BPD/8116/2002) and a grant (reference POCI/CTE-AST/56453/2004) is gratefully acknowledged.

\section{References}

Allende Prieto, C., Lambert, D. L., \& Asplund, M. 2001, ApJ, 556, L63 Alonso, A., Arribas, S., \& Martinez-Roger, C. 1996, A\&A, 313, 873 Alonso, R., Brown, T. M., Torres, G., et al. 2004, ApJ, 613, L153 Amôres, E. B., \& Lépine, J. R. D. 2005, AJ, 130, 659

Anders, E., \& Grevesse, N. 1989, Geochim. Cosmochim. Acta, 53, 197

Beirão, P., Santos, N. C., Israelian, G., \& Mayor, M. 2005, ArXiv Astrophysics e-prints

Bensby, T., Feltzing, S., \& Lundström, I. 2003, A\&A, 410, 527

Bessell, M. S., Castelli, F., \& Plez, B. 1998, A\&A, 333, 231

Bodaghee, A., Santos, N. C., Israelian, G., \& Mayor, M. 2003, A\&A, 404, 715

Bouchy, F., Pont, F., Santos, N. C., et al. 2004, A\&A, 421, L13

Bouchy, F., Pont, F., Melo, C., et al. 2005a, A\&A, 431, 1105

Bouchy, F., Udry, S., Mayor, M., et al. 2005b, A\&A, 444, L15

Brewer, M.-M., \& Carney, B. W. 2006, AJ, 131, 431

Charbonneau, D., Brown, T., Latham, D., \& Mayor, M. 2000, ApJ, 529, L45

Ecuvillon, A., Israelian, G., Santos, N. C., et al. 2004, A\&A, 426, 619

Ecuvillon, A., Israelian, G., Santos, N. C., Mayor, M., \& Gilli, G. 2006a, A\&A, 449, 809

Ecuvillon, A., Israelian, G., Santos, N. C., et al. 2006b, A\&A, 445, 633

Fischer, D. A., \& Valenti, J. 2005, ApJ, 622, 1102

Flower, P. J. 1996, ApJ, 469, 355
Fuhrmann, K. 2004, Astron. Nachr., 325, 3

Fulbright, J. P., McWilliam, A., \& Rich, R. M. 2005, Am. Astron. Soc. Meet. Abstr., 207, 133.04

Gilli, G., Israelian, G., Ecuvillon, A., Santos, N. C., \& Mayor, M. 2006, A\&A, 449,723

Gonzalez, G. 1998, A\&A, 334, 221

Grebel, E. K., \& Roberts, W. J. 1995, A\&AS, 109, 293

Guillot, T., Santos, N. C., Pont, F., et al. 2006, A\&A, in press

Henry, G. W., Marcy, G. W., Butler, R. P., \& Vogt, S. S. 2000, ApJ, 529, L41

Israelian, G., Santos, N. C., Mayor, M., \& Rebolo, R. 2001, Nature, 411, 163

Konacki, M., Torres, G., Jha, S., \& Sasselov, D. 2003, Nature, 421, 507

Konacki, M., Torres, G., Sasselov, D. D., \& Jha, S. 2005, ApJ, 624, 372

Kurucz, R. 1993, ATLAS9 Stellar Atmosphere Programs and $2 \mathrm{~km} \mathrm{~s}^{-1}$ grid. Kurucz CD-ROM No. 13, Cambridge, Mass.: Smithsonian Astrophysical Observatory, 13

Kurucz, R. L., Furenlid, I., \& Brault, J. T. L. 1984, Solar flux atlas from 296 to $1300 \mathrm{~nm}$ (National Solar Observatory Atlas, Sunspot, New Mexico: National Solar Observatory, 1984)

Lang, K. R. 1999, Astrophysical formulae, ed. K. R. Lang (New York : Springer, Astronomy and astrophysics library)

Laughlin, G., Wolf, A., Vanmunster, T., et al. 2005, ApJ, 621, 1072

Laws, C., \& Gonzalez, G. 2001, ApJ, 553, 405

Lodders, K. 2003, ApJ, 591, 1220

McCullough, P. R., Stys, J. E., Valenti, J. A., et al. 2006, ArXiv Astrophysics e-prints

Melo, C., Santos, N., Israelian, G., et al. 2006, A\&A, submitted

Murray, N., Hansen, B., Holman, M., \& Tremaine, S. 1998, Science, 279, 69

Murray, N., Paskowitz, M., \& Holman, M. 2002, ApJ, 565, 608

Nissen, P. E. 2004, in Origin and Evolution of the Elements, ed. A. McWilliam, \& M. Rauch, 154

Nordström, B., Mayor, M., Andersen, J., et al. 2004, A\&A, 418, 989

Pinsonneault, M. H., DePoy, D. L., \& Coffee, M. 2001, ApJ, 556, L59

Pont, F., Bouchy, F., Queloz, D., et al. 2004, A\&A, 426, L15

Sadakane, K., Ohkubo, M., Takeda, Y., et al. 2002, PASJ, 54, 911

Santos, N. C., Israelian, G., \& Mayor, M. 2004, A\&A, 415, 1153

Santos, N. C., Israelian, G., Mayor, M., Rebolo, R., \& Udry, S. 2003, A\&A, 398, 363

Santos, N. C., Pont, F., Melo, C., et al. 2006, ArXiv Astrophysics e-prints

Sato, B., Fischer, D. A., Henry, G. W., et al. 2005, ApJ, 633, 465

Schlegel, D. J., Finkbeiner, D. P., \& Davis, M. 1998, ApJ, 500, 525

Smith, V. V., Cunha, K., \& Lazzaro, D. 2001, AJ, 121, 3207

Sneden, C. 1973, Ph.D. Thesis, Univ. of Texas

Sozzetti, A., Yong, D., Carney, B. W., et al. 2006, AJ, 131, 2274

Takeda, Y., Sato, B., Kambe, E., et al. 2001, PASJ, 53, 1211

Udalski, A., Paczynski, B., Zebrun, K., et al. 2002, Acta Astron., 52, 1

Vauclair, S. 2004, ApJ, 605, 874 